\title{
Neuromodulation in treatment of hypertension by acupuncture: A neurophysiological prospective
}

\author{
Peyman Benharash ${ }^{1}$, Wei Zhou ${ }^{2 *}$ \\ ${ }^{1}$ Division of Cardiothoracic Surgery, University of California, Los Angeles, USA \\ ${ }^{2}$ Department of Anesthesiology, University of California, Los Angeles, USA; *Corresponding Author: wzhou@mednet.ucla.edu
}

Received 28 February 2013; revised 30 March 2013; accepted 6 April 2013

Copyright (C 2013 Peyman Benharash, Wei Zhou. This is an open access article distributed under the Creative Commons Attribution License, which permits unrestricted use, distribution, and reproduction in any medium, provided the original work is properly cited.

\section{ABSTRACT}

Hypertension is a major public health problem affecting over one billion individuals worldwide. This disease is the result of complex interactions between genetic and life-style factors and the central nervous system. Sympathetic hyperactivity has been postulated to be present in most forms of hypertension. Pharmaceutical therapy for hypertension has not been perfected, often requires a multidrug regimen, and is associated with adverse side effects. Acupuncture, a form of somatic afferent nerve stimulation has been used to treat a host of cardiovascular diseases such as hypertension. It has long been established that the two major contributors to systemic hypertension are the intrarenal reninangiotensin system and chronic activation of the sympathetic nervous system. A number of important studies on the baroreflex response and its response to acupuncture are discussed. The inhibitory effects of acupuncture on the rostral ventrolateral medulla (rVLM) reduces sympathetic nerve activity and blood pressure suggesting overactivity of the angiotensin system in this nucleus may play a role in the maintenance of hypertension. Our experimental studies have shown that electroacupuncture stimulation activates neurons in the arcuate nucleus, ventrolateral gray, and nucleus raphe to inhibit the neural activity in the rVLM in a model of visceral reflex stimulation-induced hypertension. The significant role of spinal cord opioids and nociceptin are also reviewed. Although clinical studies of acupuncture to date have met some success, they are rarely scientifically robust and do not feature randomization and long-term follow up. Based on a great deal of basic scientific evidence, large trials are desperately needed to study the effects of acupuncture on the hypertensive man.

Keywords: Central Nervous System; Electroacupuncture; Neurotransmitter; Brain Stem

\section{INTRODUCTION}

Hypertension has become a serious public health problem impacting over one billion lives worldwide [1]. At the turn of this century, 7.6 million deaths were attributable to hypertension. The majority of this disease burden occurred in working people in low to middle-income countries, while its prevalence increases with age and the lifetime risk of developing hypertension approaches $90 \%$ [2]. With the growing elderly population, dietary and life-style changes, the incidence of hypertension is expected to continue to increase.

There exist complex physiologic interactions between multiple organ systems that lead to hypertension. Genetic and environmental factors play a large role in development of this disease. Such intricacies have led clinicians to evaluate the efficacy of a wide range of therapeutics.

Current pharmacologic approaches to hypertension include agents that affect myocardial contractility and chronotropy, vascular tone, salt and water retention. All of these pharmaceuticals have numerous side effects, are costly and are often only partially effective requiring treatment with multidrug regimens.

Experimental evidence suggests that hyperactivity of the central nervous system may be involved in the pathogenesis of hypertension. For instance, individuals with high sodium intake and reduced natruresis due to high levels of renin release are prone to developing high blood pressure. The activity of the sympathetic nervous system (SNA) contributes to renin release which in turn leads to increased vascular tone as well as salt retention by increasing the levels of angiotensin and aldosterone. 
Acupuncture, a form of somatic afferent nerve stimulation, has long been postulated to exert its effects through the nervous system. Manual acupuncture and its potent alternative, electroacupuncture (EA), have been used in Asian counties to treat many cardiovascular disorders. Many physicians, however, are reluctant to use acupuncture due to its controversial benefits and largely unknown physiological mechanisms of action.

In this paper, the current body of knowledge on the effects of acupuncture on the cardiovascular system will be reviewed. We will describe the central nervous mechanisms of acupuncture and provide the rationale for the use of this technique in the treatment of hypertension.

\section{CENTRAL REGULATION OF BLOOD PRESSURE}

There is clear scientific evidence supporting the critical role of the central nervous system in the development and maintenance of hypertension. In particular, increases in SNA and derangements in arterial baroreflex function appear to contribute to the pathogenesis of this disease [3]. Development of hypertension in various animal models of hypertension, such as the spontaneously hypertensive rat (SHR), the renin transgenic (TGR mRen2) rat, the Dahl salt-sensitive rat and the dexycorticosterone acetate (DOCA)-salt rat, is associated with increased in sympathetic nerve firing. The effector mechanisms for hypertension through increased sympathetic activity include increase in vascular tone, as well as enhancement of the chronotropic and inotropic properties of the heart. The end result of such alterations is a higher than normal cardiac output and peripheral vascular resistance.

The effects of SNA on the kidneys have been elucidated and recently garnered particular clinical and therapeutic attention. Sympathetic activation stimulates renin secretion that in turn activates the renin-angiotensin-aldosternone system leading to angiotensin (Ang) II-induced vasoconstriction and sodium retention [4]. This feature leads to increased preload and therefore high cardiac-output hypertension.

Alteration of arterial baroreflex function has also been implicated in the development of hypertension [5]. Carotid sinus and aortic arch baroreceptors respond to changes in blood pressure (BP) by modulating parasysmpathetic and sympathetic outflow, thus controlling heart rate cardiac output and vasomotor tone. In response to a persistent elevation of BP, the baroreflex resets towards a higher pressure setpoint [6]. Under hypertensive conditions, resetting of the operational point of the arterial baroreflex therefore contributes to maintaining a high BP rather than opposing it. Similar to animal models of hypertension, hypertension in human subjects is associated with increases in sympathetic activity and blunted arte- rial baroreflexes [5,7-9].

Functional changes have been detected largely in hypothalamic and medullary areas that modulate sympathetic outflow [10]. Ang II contributes to cardiovascular regulation via its direct action at various hypothalamic and medullary areas to enhance sympathetic outflow, blunt the sensitivity of the baroreflex, and stimulate secretion of vasopressin [11,12].

\section{CENTRAL MECHANISM OF ACUPUNCTURE}

Over the past decade, our group and others have systematically examined the central neural regulation of visceral reflex-induced hypertension by acupuncture in different regions of brain and spinal cord. A brief description of our findings using EA will be described in the ensuing sections.

\subsection{EA Inhibition of Neural Activity in the rVLM}

The rVLM plays a critical role in the regulation of BP [13], while inhibition of neuronal function in this region results in a large drop in BP [14]. We have demonstrated that both low frequency electro- and manual-acupuncture inhibit premotor sympathetic neural firing in the rVLM and lower BP [15]. Administration of naloxone (nonspecific opioid receptor antagonist) or gabazine ( $\gamma$-aminobutyric acid or GABA type A receptor blocker) in the rVLM abolishes the EA effect, implicating the opioid receptors in this response [16]. The rVLM is an important brain stem region that processes somatic inputs during acupuncture stimulation. Electrophysiological studies of neuronal activity in the rVLM have shown that, as compared to cardiovascular inactive points (LI 6-7, G 37-39), P 5-6 and certain acupoints along the large intestine meridian (LI 4-11), located over deep somatic neural pathways (median and deep radial nerves), provide more afferent input to cardiovascular premotor sympathetic neurons in the rVLM [17]. This observation likely explains why acupuncture over these deep nerves most effectively lower elevated sympathetic outflow and BP.

\subsection{Neurotransmitters in rVLM, ARC, and vIPAG}

Investigations of EA in several early models of hypertensions suggested that release of opioids, GABA, nociceptin and serotonin in the rVLM are responsible for the BP-lowering effects of acupuncture [18-22]. More recently, we have demonstrated that the EA inhibition of visceral reflex-induced hypertension in the cat is related to the activation of $\mu$ - and $\delta$-, but not $k$-opioid receptors in the rVLM. Based on these observations, it appears that 
endorphins, enkephalins and perhaps endomorphin, but not dynorphin are mainly responsible for EA modulation of cardiovascular reflexes.

Immunohistochemical staining studies have demonstrated the presence of enkephalinergic neurons in the rVLM and endorphinergic neurons in the arcuate nucleus that project directly to the rVLM, and that both neurotransmitter systems are activated by EA [23]. EA inhibits the reflex hypertension through opioid-mediated inhibition of glutamate in the rVLM [24]. Electrophysiological studies [12] have shown that reciprocal excitatory glutamatergic (NMDA and non-NMDA) projections exist between the arcuate nucleus and vlPAG that may participate in the EA inhibition of cardiovascular function. This reciprocal projection may include a cholinergic component in the arcuate nucleus but not in the vlPAG [25].

By stimulating the presynaptic endocannabinoid CB1 receptor, EA reduces the vlPAG release of GABA but not glutamate during EA [26]. Reduced GABA disinhibits vlPAG neurons, thus increasing their activity, which, in turn, through an action in the medullary nucleus raphé pallidus (NRP) inhibits rVLM cardiovascular sympathetic neurons and related sympathoexcitatory reflex responses [27]. EA, in summary, exerts its cardiovascular effects though a complex set of excitatory and inhibitory neurotransmitters.

\subsection{Long Loop Pathway}

The hypothalamic arcuate nucleus and its interaction with the vlPAG and rVLM in the EA-cardiovascular sympathoexcitatory responses has been extensively studied $[17,21,28,29]$. When stimulated, the arcuate nucleus enhances, while its inhibition diminishes the response of vIPAG to sympathetic stimulation [21]. EA increases sympathetically-evoked discharge of vlPAG neurons, a response that can be blocked by microinjection of kainic acid (KA) into the arcuate nucleus. Additionally, EA mimics the action of excitatory amino acid DLH injection into the arcuate nucleus, and inhibits the reflex increase in BP induced by application of painful visceral stimulation. Finally, microinjection of KA into the arcuate blocks the inhibitory influence of EA on reflex hypertension. These results suggest that excitatory projections from the arcuate nucleus to the vlPAG appear to be essential to the inhibitory influence of EA on the reflex increase in BP induced by painful afferent stimulation.

Direct axonal projections from the vlPAG to the medulla have been documented in tract tracing studies [30]. Inhibitory inputs from the vlPAG to premotor sympathetic neurons in the rVLM reduce sympathetic outflow and reflex elevations in BP [29]. However, a vlPAG projection to the raphé, in particular the nucleus raphé obscurus (NRO) also exists and has been thought to form an indirect pathway from the vlPAG to the rVLM that is involved in the EA-cardiovascular response [31]. Recent studies have suggested that the NRP, located more ventrally than the NRO or the nucleus raphé magnus, contains more cells that are activated during median nerve stimulation with EA at the P 5-6 acupoints [32]. Chemical blockade of the NRP with KA transiently reverses activation of neurons in the rVLM during stimulation of the vlPAG as well as EA modulation of visceral excitatory reflexes [33].

Serotonergic projections from the raphé acting on $5-\mathrm{HT}_{1 \mathrm{~A}}$ receptors in the rVLM complete the vlPAGNRP-rVLM circuit for cardiovascular reactivity [33]. Such connections between the NRP and the rVLM play an important role in the long-loop modulation of cardiovascular sympathetic outflow during reflex visceral stimulation. Whether direct or not, projections from the vlPAG to the rVLM provide an important source for the inhibitory influence of EA on rVLM premotor neurons and ultimately curb the sympathoexcitatory cardiovascular response [31].

\subsection{Spinal Cord and EA in Cardiovascular Reactivity}

The spinal cord is the primary center for processing of somatic and visceral inputs as well as generation of outputs to effector organs involved in cardiovascular reflex regulation [34]. Anatomical and physiological studies indicate that the dorsal horn of the spinal cord serves as a major center for EA-induced analgesia [35,36]. Since opioid or nociceptin-like immunoreactivity is present in the spinal sympathetic nuclei (i.e., intermediolateral column, IML) [37,38], it is possible that EA also influences neurotransmission between the brain stem and the IML [39]. We have demonstrated that both opioids and nociceptin, reduce the response to rVLM-induced sympathoexcitation, indicating that the two peptides can regulate sympathetic outflow [40,41]. Additionally, there has been a suggestion that descending pathways from the brain stem may influence the segmental processing of somatic inputs during EA [42,43]. Afferent stimulation can modulate sympathetic activity through the inhibition of excitatory interneurons [44]. Furthermore, somatic stimulation can elicit excitatory and inhibitory responses in both IML and dor sal horn interneurons, depending on the dermatome stimulated [45]. These interneurons appear to form important links in the spinal cord circuitry involved in autonomic control [46]. These findings implicate opioids and nociceptin as the central mediators of the cardiovascular response within the spinal cord, both by intrinsic action on the cord and by modulation of brainstem signals to the spinal results indicate that opioid and nociceptin play a role in the processing of spinal 
cord interneuron activity in the EA response. However, spinal circuits modulating cardiovascular reactivity during acupuncture require further elucidation.

\section{ACUPOINT SELECTION}

We have previously evaluated the point specificity in EA treatment of reflex-induced hypertension in animals. Distention of gallbladder or other viscera leads to stimulation of the sympathetic nervous system through activation of cardiovascular premotor sympathetic neurons in the rVLM. We observed that EA at P 5-6 (pericardial meridian, overlying the median nerve) and LI 10-11 (large intestine meridian, overlying the deep radial nerve) are most effective in reducing reflex-induced hypertension. EA at LI 4-L7 (large intestine and lung meridians, overlying branches of median and the superficial radial nerve) and S 36-37 (stomach meridian overlying the deep peroneal nerve) are less effective, while EA at LI 6-7 and K1-B67 do not have any impact on BP. Furthermore, direct stimulation of the deep or superficial nerves underneath the acupoints produces similar results $[17,47]$. Similar observations have been made in a rat model employing gastric distension to elevate BP $[15,48]$.

\section{STIMULATION PARAMETERS}

EA has preferentially been used in scientific studies on cardiovascular diseases due to the ability to precisely control stimulation parameters; outcomes from manual acupuncture are operator dependent therefore not as reproducible. A low frequency of $2 \mathrm{~Hz}$ is used more frequently to treat hypertension because EA induces frequency-dependent release of neuropeptides. Experimentally, stimulation at $2 \mathrm{~Hz}$ produces a significant increase in enkephalin-like immunoreactivity but not in dynorphin immunoreactivity, whereas $100 \mathrm{~Hz}$ increases dynorphin immunoreactivity but not enkephalin immunoreactivity [49]. Similar results were confirmed in humans [50]. In the brain, enkephalins and endorphins as well as their associated $\delta$ - and $\mu$-opioid receptors have been shown to be more important in modulating the cardiovascular actions of EA than dynorphin ( $\kappa$-opioid) [51].

In our rat model of reflex hypertension, sham acupuncture involving needle insertion without manipulation at P 5-6 or LI 6-7 acupoints did not attenuate the gastric distention-induced hypertension, thus demonstrating that this procedure can serve as a control for EA. However, EA at P 5-6, H 6-7 (overlying the ulnar nerve) or S 36-37 with low current $(2 \mathrm{~mA}$ ) and low frequency $(2 \mathrm{~Hz})$ for 30 min inhibited reflex-induced hypertension. Increasing the stimulation frequency to 40 or $100 \mathrm{~Hz}$ did not inhibit the elevated BP. In this regard, we observed a reciprocal relationship between the frequency of stimulation and the afferent response. Thus, it appears that low frequency, low current EA in a point specific manner optimally influences reflex-induced hypertension $[15,48]$.

\section{CLINICAL STUDY OF EA IN TREATMENT OF HYPERTENSION}

Clinical studies on the effectiveness of acupuncture at specific acupoints to in essential hypertension have been reported on for over half a century. As early as the 1950s, publications in China reported that acupuncture effectively reduced BP in hypertensive patients [52,53]. In 1975, Tam et al., found that acupuncture produced a significant reduction in systolic and diastolic BP in 24 out of 28 patients with essential hypertension [54]. Figure 1 shows a number of clinically-utilized acupoints found to be effective in reducing BP, including Pericardium 5, 6 (P 5, 6), Stomach 36 (ST 36), Large Intestine 4, 11 (LI 4, 11), Bladder 18, 20 (BL 18, 20), Gallbladder 34 (GB 34) $[55,56]$.

Despite the suggested beneficial effects of acupuncture on hypertension, there have been many weaknesses in prior acupuncture clinical research. For example, the sample sizes are typically quite small and power tests to determine sample sizes have not been calculated a priori; randomization has been rare, and the studies have almost uniformly lacked adequate control groups and control acupoints. The period of follow-up after treatment has generally been inadequate or unspecified. Many studies have employed traditional Chinese medicine theory, including applying the meridian hypothesis and Qi theory in place of modern Western scientific principles underlying neuroscience and cardiovascular physiology. Importantly, few studies utilize EA, which is easier to control and to standardize stimulation parameters. In this regard, several recent review publications have expressed concern regarding the lack of controls and the potential for placebo effects in prior studies, and have made several pertinent suggestions for improving future studies [57]. Improvements for future study of acupuncture in hypertension include 24-hour ambulatory BP monitoring to obtain a more accurate Snapshot [58]. Carefully constructed and adequately powered studies need to be performed on to evaluate the efficacy of acupuncture in chronic maintenance of BP reduction, as well as the frequency of treatment applications needed to sustain this effect.

\section{SUMMARY}

Hypertension is a major cardiovascular disease and poses serious health risks and mortality to a large number of patients. Current available pharmacologic therapies are somewhat effective and most patients require multiple drug regimens. Hypertension likely has a complex central mechanism that involves increased sympathetic 
activity and resetting of the baroreceptor setpoints. Therefore, central neuromodulation seems to be a rational therapy for this problem. Acupuncture is a powerful tool in neuromodulation and its effects on the central nervous system and hypertension have been studies. Acupuncture has been shown to decrease BP in hypertensive patients and in animal models of hypertension. The mechanisms underlying the beneficial effects of acupuncture are associated with modulation of sympathetic outflow and possibly endocrine system. Experimental studies have shown that EA inhibits the reflex-induced hypertension by modulating the activity of cardiovascular presympa- thetic neurons in the rVLM. Activation of neurons in the arcuate nucleus of the hypothalamus, vlPAG in the midbrain and NRP in the medulla by EA can inhibit the activity of premotor sympathetic neurons in the rVLM. Glutamate, acetylcholine, opioids, GABA, nociceptin, serotonin, NO, endocannabinoids in the brain all appear to participate in the EA antihypertensive response (Figure 2).

Clinical trials of EA in hypertensive subjects have reported favorable results over the past few decades. Studies with large sample size and further mechanistic studies are recommended in order to expand the role of this
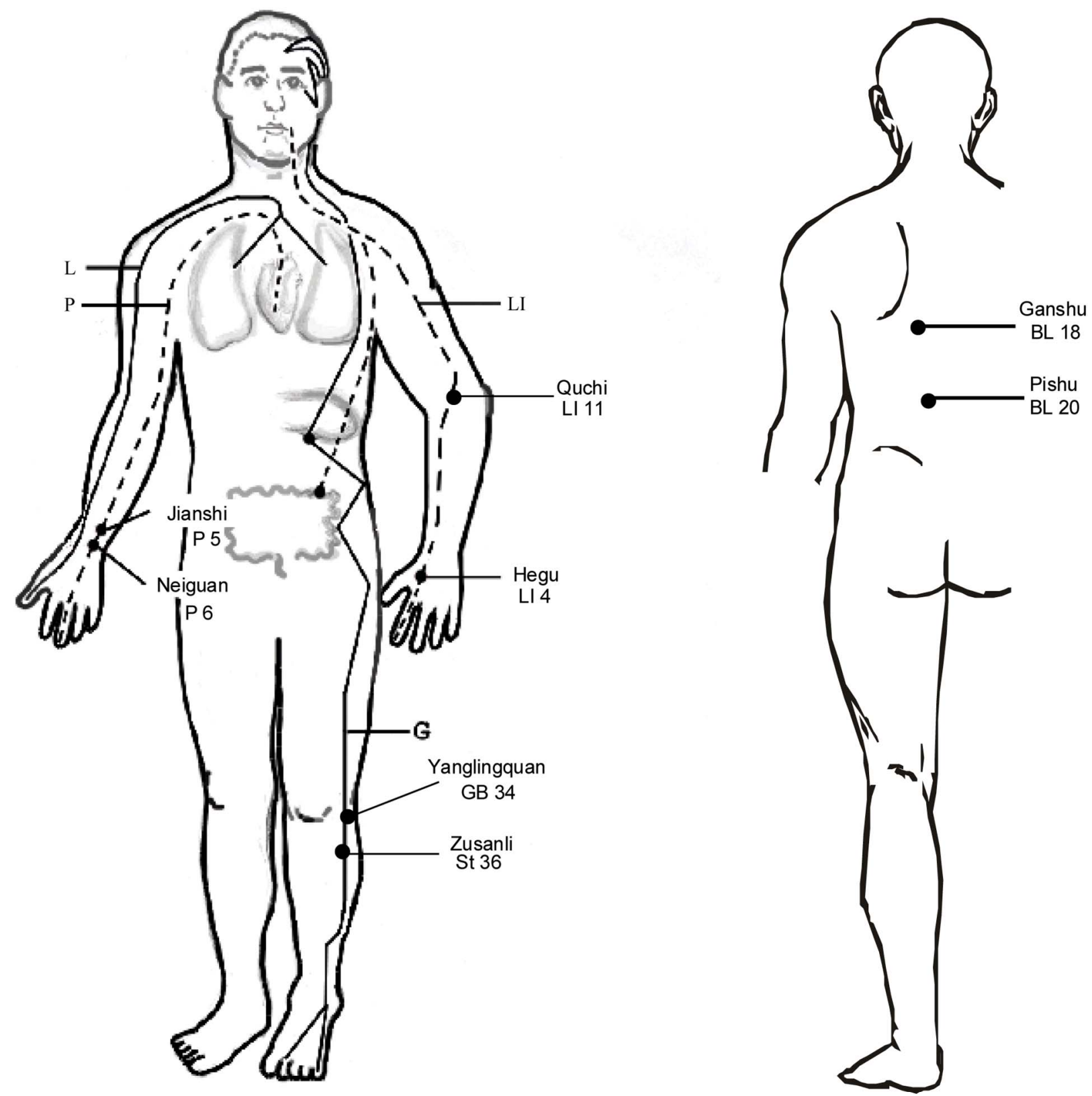

Figure 1. Location of acupoints along meridians. Note, although all meridians are bilateral, they are only drawn on one side for simplicity. Abbreviations of meridians: G: gallbladder; L: lung; LI: large intestine; P: pericardium. 


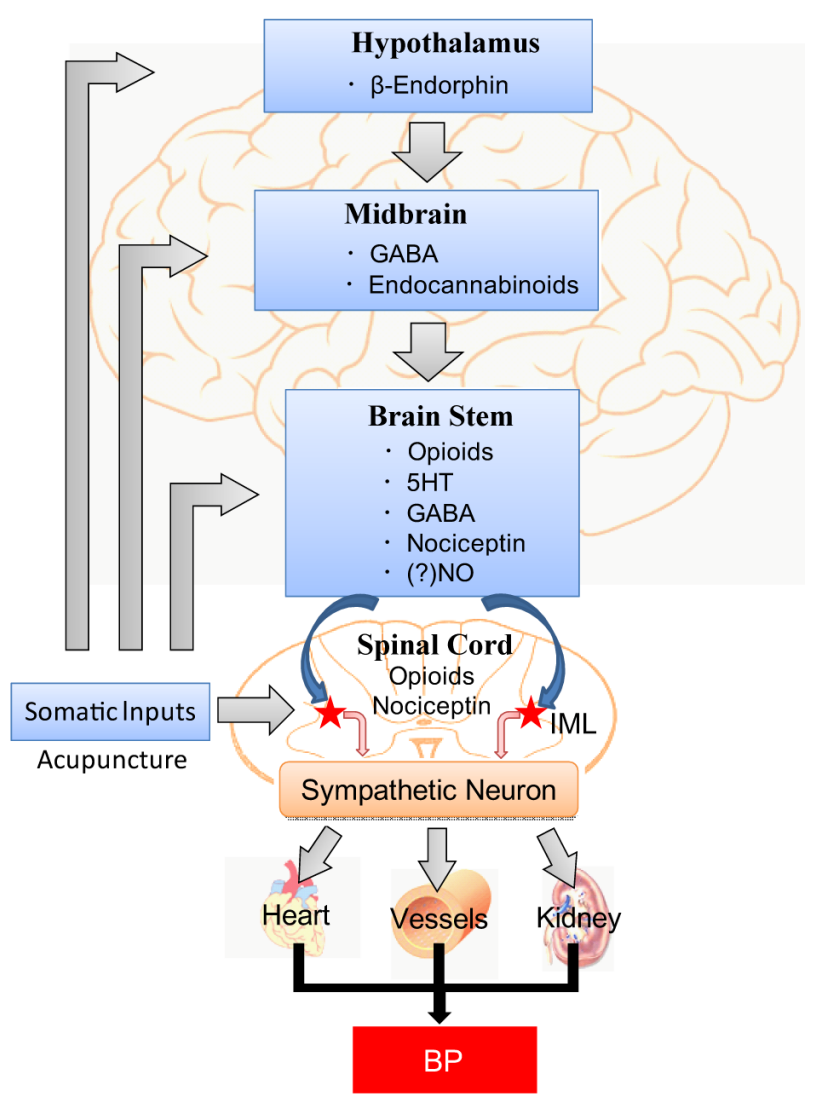

Figure 2. Central modulation of blood pressure by acupuncture. Abbreviations: GABA, $\gamma$-aminobutyric acid; 5HT, 5-hydroxytryptamine or serotonin; NO, nitric oxide; IML, intermediolateral column of the spinal cord (from Zhou, W., Longhurst, J.C., Evidence-Based Complementary and Alternative Medicine, 2012).

powerful therapy in treatment of cardiovascular diseases in man.

\section{REFERENCES}

[1] Hajjar, I. and Kotchen, T.A. (2003) Trends in prevalence, awareness, treatment, and control of hypertension in the United States, 1988-2000. JAMA, 290, 199-206. doi:10.1001/jama.290.2.199

[2] Vasan, R.S., Beiser, A., Seshadri, S., et al. (2002) Residual lifetime risk for developing hypertension in middleaged women and men: The Framingham heart study. JAMA, 287, 1003-1010. doi:10.1001/jama.287.8.1003

[3] Veerasingham, S.J. and Raizada, M.K. (2003) Brain reninangiotensin system dysfunction in hypertension: Recent advances and perspectives. British Journal of Pharmacology, 139, 191-202. doi:10.1038/sj.bjp.0705262

[4] Zheng, H., Li, Y.F., Wang, W. and Patel, K.P. (2009) Enhanced angiotensin-mediated excitation of renal sympathetic nerve activity within the paraventricular nucleus of anesthetized rats with heart failure. American Journal of Physiology-Regulatory, Integrative and Comparative Physiology, 297, R1364-R1374. doi:10.1152/ajpregu.00149.2009
[5] Matsukawa, T., Gotoh, E., Hasegawa, O., et al. (1991) Reduced arterial baroreflex control of muscle sympathetic nerve activity in young borderline hypertensives. Functional Neurology, 6, 113-120.

[6] Andresen, M.C. and Yang, M. (1989) Arterial baroreceptor resetting: Contributions of chronic and acute processes. Clinical and Experimental Pharmacology \& Physiology, Supplement, 15, 19-30. doi:10.1111/j.1440-1681.1989.tb02993.x

[7] Esler, M., Rumantir, M., Kaye, D. and Lambert, G. (2001) The sympathetic neurobiology of essential hypertension: Disparate influences of obesity, stress, and noradrenaline transporter dysfunction? American Journal of Hypertension, 14, 139S-146S. doi:10.1016/S0895-7061(01)02081-7

[8] Goldstein, D.S. (1981) Plasma norepinephrine during stress in essential hypertension. Hypertension, 3, 551-556. doi:10.1161/01.HYP.3.5.551

[9] Schultz, H.D., Li, Y.L. and Ding, Y. (2007) Arterial chemoreceptors and sympathetic nerve activity: Implications for hypertension and heart failure. Hypertension, 50, 613. doi:10.1161/HYPERTENSIONAHA.106.076083

[10] Colombari, E., Sato, M.A, Cravo, S.L., Bergamaschi, C.T., Campos, R.R. and Lopes, O.U. (2001) Role of the medulla oblongata in hypertension. Hypertension, 38, 549-554. doi:10.1161/01.HYP.38.3.549

[11] Averill, D.B. and Diz, D.I. (2000) Angiotensin peptides and baroreflex control of sympathetic outflow: Pathways and mechanisms of the medulla oblongata. Brain Research Bulletin, 51, 119-128. doi:10.1016/S0361-9230(99)00237-3

[12] Dampney, R.A., Fontes, M.A., Hirooka, Y., Horiuchi, J., Potts, P.D. and Tagawa, T. (2002) Role of angiotensin II receptors in the regulation of vasomotor neurons in the ventrolateral medulla. Clinical and Experimental Pharmacology and Physiology, 29, 467-472. doi:10.1046/j.1440-1681.2002.03658.x

[13] Guyenet, P. (1990) Role of ventral medulla oblongata in blood pressure regulation. In: Spyer, K. and Loewy, A.D., Eds., Central Regulation of Autonomic Functions, Oxford University Press, Oxford, 145-167.

[14] Guertzenstein, P.G. and Silver, A. (1974) Fall in blood pressure produced from discrete regions of the ventral surface of the medulla by glycine and lesions. Journal of Physiology, 242, 489-503.

[15] Zhou, W., Tjen-A-Looi, S.C. and Longhurst, J.C. (2005) Brain stem mechanisms underlying acupuncture modality-related modulation of cardiovascular responses in rats. Journal of Applied Physiology, 99, 851-860. doi:10.1152/japplphysiol.01365.2004

[16] Tjen-A-Looi, S.C., Li, P. and Longhurst, J.C. (2003) Prolonged inhibition of rostral ventral lateral medullary premotor sympathetic neurons by electroacupuncture in cats. Autonomic Neuroscience, 106, 119-131. doi:10.1016/S1566-0702(03)00076-6

[17] Tjen-A-Looi, S.C., Li, P. and Longhurst, J.C. (2004) Medullary substrate and differential cardiovascular responses during stimulation of specific acupoints. American Journal of Physiology-Regulatory, Integrative and Compara- 
tive Physiology, 287, R852-R862. doi:10.1152/ajpregu.00262.2004

[18] Crisostomo, M.M., Li, P., Tjen-A-Looi, S.C. and Longhurst, J.C. (2005) Nociceptin in rVLM mediates electroacupuncture inhibition of cardiovascular reflex excitatory response in rats. Journal of Applied Physiology, 98, 20562063. doi:10.1152/japplphysiol.01282.2004

[19] Huangfu, D.H. and Li, P. (1988) Role of nucleus raphe obscurus in the inhibition of defense reaction by deep peroneal nerve stimulation. Chinese Journal of Physiology Science, 4, 77-83.

[20] Huangfu, D.H. and Li, P. (1988) The inhibitory effect of ARC-PAG-NRO system on the ventrolateral medullary neurons in the rabbit. Chinese Journal of Physiology Science, 4, 115-125.

[21] Li, P., Tjen-A-Looi, S.C. and Longhurst, J.C. (2006) Excitatory projections from arcuate nucleus to ventrolateral periaqueductal gray in electroacupuncture inhibition of cardiovascular reflexes. American Journal of Physiology -Heart and Circulatory Physiology, 290, H2535-H2542. doi:10.1152/ajpheart.00972.2005

[22] Li, P. and Yao, T. (1992) Mechanism of the modulatory effect of acupuncture on abnormal cardiovascular functions. Shanghai Medical University, Shanghai.

[23] Guo, Z.L. and Longhurst, J.C. (2007) Expression of c-Fos in arcuate nucleus induced by electroacupuncture: Relations to neurons containing opioids and glutamate. Brain Research, 1166, 65-76. doi:10.1016/j.brainres.2007.06.042

[24] Zhou, W., Fu, L.W., Guo, Z.L. and Longhurst, J.C. (2007) Role of glutamate in the rostral ventrolateral medulla in acupuncture-related modulation of visceral reflex sympathoexcitation. American Journal of Physiology-Heart and Circulatory Physiology, 292, H1868-H1875. doi:10.1152/ajpheart.00875.2006

[25] Dampney, R.A., Horiuchi, J., Tagawa, T., Fontes, M.A., Potts, P.D. and Polson, J.W. (2003) Medullary and supramedullary mechanisms regulating sympathetic vasomotor tone. Acta Physiologica Scandinavica, 177, 209218. doi:10.1046/j.1365-201X.2003.01070.X

[26] Fu, L.W. and Longhurst, J.C. (2009) Electroacupuncture modulates vlPAG release of GABA through presynaptic cannabinoid CB1 receptors. Journal of Applied Physiology, 106, 1800-1809. doi:10.1152/japplphysiol.91648.2008

[27] Tjen-A-Looi, S.C., Li, P. and Longhurst, J.C. (2009) Processing cardiovascular information in the vlPAG during electroacupuncture in rats: Roles of endocannabinoids and GABA. Journal of Applied Physiology, 106, 17931799. doi:10.1152/japplphysiol.00142.2009

[28] Li, M., Tjen-A-Looi, S.C. and Longhurst, J.C. (2010) Electroacupuncture enhances preproenkephalin mRNA expression in rostral ventrolateral medulla of rats. Neuroscience Letters, 477, 61-65. doi:10.1016/j.neulet.2010.04.025

[29] Tjen-A-Looi, S.C., Li, P. and Longhurst, J.C. (2006) Midbrain vlPAG inhibits rVLM cardiovascular sympathoexcitatory responses during electroacupuncture. American Journal of Physiology-Heart and Circulatory Physiol- ogy, 290, H2543-H2553. doi:10.1152/ajpheart.01329.2005

[30] Loewy, A. (1990) Central autonomic pathways. In: Spyer, K. and Loewy, A.D., Eds., Central Regulation of Autonomic Functions, Oxford University Press, New York, 88103.

[31] Li, P., Tjen-A-Looi, S.C., Guo, Z.L., Fu, L.W. and Longhurst, J.C. (2009) Long-loop pathways in cardiovascular electroacupuncture responses. Journal of Applied Physiology, 106, 620-630.

doi:10.1152/japplphysiol.91277.2008

[32] Guo, Z.L., Moazzami, A.R., Tjen-A-Looi, S.C. and Longhurst, J.C. (2008) Responses of opioid and serotonin containing medullary raphe neurons to electroacupuncture. Brain Research, 1229, 125-136. doi:10.1016/j.brainres.2008.07.020

[33] Li, P., Tjen-A-Looi, S.C. and Longhurst, J.C. (2010) Nucleus raphe pallidus participates in midbrain-medullary cardiovascular sympathoinhibition during electroacupuncture. American Journal of Physiology-Regulatory, Integrative and Comparative Physiology, 299, R1369-R1376. doi:10.1152/ajpregu.00361.2010

[34] Longhurst, J.C. (2003) Neural regulation of the cardiovascular system. In: Al, S.E., Ed., Fundamental Neuroscience, 2nd Edition, Academic Press, San Diego, 935966.

[35] Lee, J.H. and Beitz, A.J. (1992) Electroacupuncture modifies the expression of c-fos in the spinal cord induced by noxious stimulation. Brain Research, 577, 8091. doi:10.1016/0006-8993(92)90540-P

[36] Lee, J.H. and Beitz, A.J. (1993) The distribution of brainstem and spinal cord nuclei associated with different frequencies of electroacupuncture analgesia. Pain, 52, 11-28. doi:10.1016/0304-3959(93)90109-3

[37] Dun, N.J., Dun, S.L. and Hwang, L.L. (1997) Nociceptinlike immunoreactivity in autonomic nuclei of the rat spinal cord. Neuroscience Letters, 234, 95-98. doi:10.1016/S0304-3940(97)00666-6

[38] Quirion, R. (1984) Pain, nociception and spinal opioid receptors. Progress in Neuro-Psychopharmacology \& Biological Psychiatry, 8, 571-579. doi:10.1016/0278-5846(84)90017-4

[39] Hofstetter, C.P., Card, J.P. and Olson, L. (2005) A spinal cord pathway connecting primary afferents to the segmental sympathetic outflow system. Experimental Neurology, 194, 128-138. doi:10.1016/j.expneurol.2005.01.028

[40] Zhou, W., Hsiao, I., Lin, V.W. and Longhurst, J.C. (2006) Modulation of cardiovascular excitatory responses in rats by transcutaneous magnetic stimulation: Role of the spinal cord. Journal of Applied Physiology, 100, 926-932. doi:10.1152/japplphysiol.01130.2005

[41] Zhou, W., Mahajan, A. and Longhurst, J.C. (2009) Spinal nociceptin mediates electroacupuncture-related modulation of visceral sympathoexcitatory reflex responses in rats. American Journal of Physiology: Heart and Circulatory Physiology, 297, H859-H865. doi:10.1152/ajpheart.00149.2009

[42] Han, J.S., Xie, G.X., Zhou, Z.F., Folkesson, R. and Teren- 
ius, L. (1982) Enkephalin and beta-endorphin as mediators of electroacupuncuture analgesia in rabbits: An antiserum microinjection study. In: Trabucchi, E.C.A.M., Ed., Regulatory Peptides, Raven Press, New York, 369377.

[43] Pomeranz, B. and Cheng, R. (1979) Suppression of noxious responses in single neurons of cat spinal cord by electroacupuncture and its reversal by the opiate antagonist naloxone. Experimental Neurology, 64, 327-341. doi:10.1016/0014-4886(79)90273-5

[44] Wyszogrodski, I. and Polosa, C. (1973) The inhibition of sympathetic preganglionic neurons by somatic afferents. Canadian Journal of Physiology and Pharmacology, 51, 29-38. doi:10.1139/y73-005

[45] Chau, D., Johns, D.G. and Schramm, L.P. (2000) Ongoing and stimulus-evoked activity of sympathetically correlated neurons in the intermediate zone and dorsal horn of acutely spinalized rats. Journal of Neurophysiology, 83, 2699-2707.

[46] Deuchars, S.A. (2007) Multi-tasking in the spinal cord -Do "sympathetic" interneurones work harder than we give them credit for? The Journal of Physiology, 580, 723-729. doi:10.1113/jphysiol.2007.129429

[47] Li, P., Pitsillides, K.F., Rendig, S.V., Pan, H.L. and Longhurst, J.C. (1998) Reversal of reflex-induced myocardial ischemia by median nerve stimulation: A feline model of electroacupuncture. Circulation, 97, 1186-1194. doi:10.1161/01.CIR.97.12.1186

[48] Zhou, W., Fu, L.W., Tjen-A-Looi, S.C., Li, P. and Longhurst, J.C. (2005) Afferent mechanisms underlying stimulation modality-related modulation of acupuncture-related cardiovascular responses. Journal of Applied Physiology, 98, 872-880. doi:10.1152/japplphysiol.01079.2004

[49] Han, J.S. (2003) Acupuncture: Neuropeptide release produced by electrical stimulation of different frequencies. Trends in Neurosciences, 26, 17-22. doi:10.1016/S0166-2236(02)00006-1
[50] Han, J.S., Chen, X.H., Sun, S.L., et al. (1991) Effect of low- and high-frequency TENS on Met-enkephalin-ArgPhe and dynorphin A immunoreactivity in human lumbar CSF. Pain, 47, 295-298. doi:10.1016/0304-3959(91)90218-M

[51] Li, P., Tjen-A-Looi, S.C. and Longhurst, J.C. (2001) Rostral ventrolateral medullary opioid receptor subtypes in the inhibitory effect of electroacupuncture on reflex autonomic response in cats. Autonomic Neuroscience: Basic \& Clinical, 89, 38-47. doi:10.1016/S1566-0702(01)00247-8

[52] Acupuncture Research Group of Anhui University (1961) Primary observation of 179 hypertensive cases treated with acupuncture. Acta Acad. Med. An Hui, 4, 6.

[53] Zhang, C.L. (1956) Clinical investigation of acupuncture therapy. Clinic Journal of Medicine, 42, 514-517.

[54] Tam, K.-C. and Yiu, H.-H. (1975) The effect of acupuncture on essential hypertension. The American Journal of Chinese Medicine, 3, 369-375. doi:10.1142/S0192415X7500044X

[55] Li, P., Ayannusi, O., Reid, C. and Longhurst, J.C. (2004) Inhibitory effect of electroacupuncture (EA) on the pressor response induced by exercise stress. Clinical Autonomic Research, 14, 182-188. doi:10.1007/s10286-004-0175-1

[56] Qi, L.Z. (1994) Recent advance in the study of theraputic effect on hypertension by acupuncture and moxibustion. Shanghai Journal of Acupuncture and Moxibustion, 13, 87-89.

[57] Streitberger, K. and Kleinhenz, J. (1998) Introducing a placebo needle into acupuncture research. Lancet, 352, 364-365. doi:10.1016/S0140-6736(97)10471-8

[58] Jacobsson, F., Himmelmann, A., Bergbrant, A., Svensson, A. and Mannheimer, C. (2000) The effect of transcutaneous electric nerve stimulation in patients with therapyresistant hypertension. Journal of Human Hypertension, 14, 795-798. doi:10.1038/sj.jhh.1001112

NRP: Medullary nucleus raph'e pallidus

NRO: Nucleus raph'e obscurus

IML: Intermediolateral column

GABA: $\gamma$-aminobutyric acid

nNOS: Neuronal nitric oxide synthase

KA: Kainic acid 